\title{
Normas de DeRechos Humanos: enTRE PRINCIPIOS Y REGLAS ${ }^{1}$ Human Rights Norms: Between Principles and Rules
}

\author{
Julieta A. RABANOS ${ }^{2}$ \\ Università degli Studi di Genova, Génova, Italia
}

\section{Resumen}

En ocasión del $70^{\circ}$ aniversario de la Declaración Universal de Derechos Humanos (DUDH), propongo reflexionar sobre el problema conceptual relacionado a las disposiciones normativas que son usadas para reconocer y/o establecer derechos humanos. La pregunta puede ser formulada así: estas disposiciones normativas, ¿qué tipo de normas expresan? ¿Son reglas, principios o ambos, de acuerdo con las circunstancias? Responder a esta pregunta implica la solución de dos problemas diferentes. Por una parte, un problema conceptual: ¿qué tipo de teoría o enfoque puede dar mejor cuenta de las normas de derechos humanos? Por otra parte, un problema axiológico: ¿cómo deberíamos considerar a las normas de derechos humanos, de acuerdo con una determinada (correcta, mejor) doctrina o ideología? En este artículo, intento explorar y ofrecer algunas respuestas a esta pregunta central y a sus problemas conectados.

\section{Palabras clave}

Derechos humanos, normas y disposiciones normativas, reglas jurídicas, principios jurídicos, interpretación jurídica.

\begin{abstract}
On the occasion of the 70th anniversary of the Universal Declaration of Human Rights (UDHR), I propose to reflect about the conceptual problem regarding the normative texts or sentences that are used to recognise and/or establish human rights. The question can be put in these terms: what kind of norms do these normative texts or sentences express? Are they rules, principles, or both, depending on the circumstances? Answering this question involves the solution of two distinct problems. On the one hand, a conceptual problem: what kind of theory or approach can give better account of human rights norms? On the other hand, an axiological problem: how should we consider human right norms, according a given (best, correct) doctrine or ideology? In this article, I try to explore and offer some answers to this main question and its connected problems.
\end{abstract}

\section{Keywords}

Human Rights, Norms and Normative Texts or Sentences, Legal Rules, Legal Principles, Legal Interpretation.

\footnotetext{
${ }^{1}$ Este artículo fue publicado originalmente en italiano, en P. Ivaldi, L. Schiano di Pepe (eds.), I diritti umani settant'anni dopo. L'attualità della Dichiarazione universale tra questioni irrisolte e nuove minacce, Genova: GUP, 2019, pp. 33-43. Versión traducida por la autora.

${ }^{2}$ Investigadora postdoctoral en filosofía del derecho del Departamento de Derecho de la Universidad de Génova (Istituto Tarello per la filosofía del diritto), Italia. Correo electrónico: julieta.rabanos@giuri.unige.it; ORCID: https:/orcid. org/0000-0003-0490-3779.
} 


\section{Introducción}

Los setenta años de la Declaración Universal de Derechos Humanos (1948), aniversario celebrado el pasado 10 en diciembre de 2018, son sin duda una ocasión importante para reflexionar acerca de los derechos humanos.

En este breve artículo, propongo una reflexión acerca del problema conceptual relativo a las disposiciones normativas que son utilizadas para reconocer y/o establecer ${ }^{3}$ derechos humanos. La pregunta puede ser formulada en estos términos: ¿qué tipo de normas expresan estas disposiciones? ¿Son reglas, principios o bien ambos, dependiendo de las circunstancias? ? $^{4}$

La respuesta a esta pregunta implica dar solución a dos problemas diferentes. Por un lado, un problema conceptual: ¿qué tipo de teoría o de enfoque puede dar mejor cuenta de las normas de derechos humanos? ¿Cuál teoría o enfoque se adecúa mejor a su estructura, a sus condiciones, ${ }^{5}$ etc.? Por otro lado, un problema axiológico: ¿cómo deberíamos considerar a las normas de derechos humanos, a la luz de una determinada doctrina o ideología considerada la mejor o la más correcta?

En consideración de lo anterior, la estructura de este ensayo será la siguiente. En la sección 2 se efectuarán algunas distinciones conceptuales preliminares, necesarias para el desarrollo de la temática en objeto. La sección 3 ofrece una respuesta a la pregunta acerca de qué tipo de normas expresan las disposiciones normativas utilizadas para reconocer y/o establecer derechos humanos, tanto desde el punto de vista conceptual (punto 3.1) como desde el punto de vista axiológico (punto 3.2). La sección 4 está dedicada al modo en el cual sería posible resolver las tensiones identificadas en la sección precedente. Finalmente, la sección 5 contiene una breve conclusión acerca de las reflexiones realizadas en las secciones anteriores.

Una aclaración preliminar: en el ámbito de este ensayo, las nociones de 'derechos humanos' y 'derechos fundamentales' serán consideradas como expresiones sinónimas: por tanto, toda reflexión sobre 'derechos fundamentales' se entiende también hecha sobre 'derechos humanos', y viceversa.

\section{Algunas distinciones conceptuales preliminares}

\subsection{Disposición normativa y norma}

En el lenguaje de los juristas, a menudo se usa el término 'norma' para hacer referencia tanto a los enunciados de las fuentes del derecho como al significado de estos (Guastini, 2018, p. 77).

Sin embargo, enunciados y significados son dos cosas que bien pueden ser distinguidas. A tal fin, siguiendo a Guastini, “(i) denominaremos 'disposición' a todo enunciado normativo contenido en una fuente del derecho; y (ii) denominaremos 'norma' no al enunciado mismo, sino a su contenido de significado" (2018, p. 78). El pasaje de una disposición a una norma se realiza a través de la interpretación, siendo así 'norma' una "disposición interpretada y por tanto reformulada" (Guastini, 2018, p. 80).

\footnotetext{
${ }^{3}$ En el presente trabajo, no deseo tomar posición en el debate acerca del estatus de los derechos humanos, como sería afirmar que estos preexisten a la promulgación de un corpus normativo que los contenga (viendo, así, a esta promulgación como un reconocimiento o descubrimiento de un quid preexistente, y no como creación) o afirmar que estos existen solo por el hecho de haber sido promulgados (viendo, así, la promulgación como la fuente de la creación de los derechos ex nihilo).

${ }^{4}$ Es decir, según la interpretación dada a cada disposición, en sentido amplio, por el intérprete. Véase el punto 2 del presente trabajo.

${ }^{5}$ Incluso si la pregunta se formula en estos términos, de todas formas, es cierto que tanto la estructura como las condiciones dependen de la teoría elegida. En el presente trabajo, he decidido formular una pregunta en estos términos por motivos de brevedad.
} 
Las consideraciones anteriores nos permiten, en resumen, decir que: i) puede no existir una relación unívoca entre disposición y norma; ${ }^{6}$ ii) una misma disposición puede expresar más de una norma (conjunta o disyuntamente); y iii) no se puede hablar de normas sino como producto de una actividad interpretativa (Guastini, 2018, p. 80). ${ }^{7}$

\subsection{Principios y reglas: sobre su posible diferenciación}

Preguntarse si una norma es un principio y/o una regla presupone: i) sostener que tanto los principios como las reglas constituyen dos tipos de normas (Guastini, 2018, pp. 209-ss); ${ }^{8}$ y ii.1) sostener que existe una diferencia entre principios y reglas; $y$, en consecuencia, ii.2) rechazar la posición según la cual se niega que exista una diferencia tal (la así llamada tesis de la no diferenciación). ${ }^{?}$

En relación con la primera presuposición, hay un acuerdo general entre teóricos y juristas. No así, sin embargo, en relación con la segunda presuposición, principalmente en cuanto esta "distinción depende, evidentemente, de los conceptos empleados" y subsiste una pluralidad de definiciones diferentes sostenidas por distintos autores y juristas (Guastini, 2018, p. 208).

A grandes rasgos, entre aquellos que aceptan estos dos presupuestos, pueden identificarse dos tesis generalmente sostenidas: i) la tesis de la diferenciación fuerte, en adelante TDF (según la cual la diferencia entre reglas y principios es de tipo cualitativo y estructural); y ii) la tesis de la diferenciación débil, en adelante TDD (según la cual la diferencia es de tipo cuantitativo y gradual). ${ }^{10}$

\subsubsection{La TDF}

Para los autores que sostienen la TDF, los principios y las reglas son ontológicamente diferentes: son entidades cuyas características esenciales no coinciden, y que funcionan de modo diferente en el marco de la dinámica jurídica y del razonamiento jurídico (Dworkin, 1989, pp. 61-ss). En general, reconstruyen las diferencias entre principios y reglas en relación con:

1) El modo en el cual estos regulan las conductas ${ }^{11}$ o son aplicados; ${ }^{12}$

2) En modo en el cual se determina su pertenencia a un sistema jurídico; ${ }^{13} y$

3) El modo en el cual se resuelven los conflictos entre estos (regla-regla y principio-principio). ${ }^{14}$

\footnotetext{
${ }^{6}$ El modo de entender esta relación depende de la teoría de la interpretación escogida y hecha propia. Cfr. por ejemplo, (Guastini, 2018, pp. 497-ss).

${ }^{7}$ Aquí, la actividad interpretativa es considerada como una actividad de decisión sobre el significado, cuyo objeto es una disposición y cuyo resultado es una norma (Guastini, 2018, capítulo I). Sin embargo, según la teoría de la interpretación elegida, la actividad de interpretación puede ser considerada una actividad decisoria o una actividad de descubrimiento o conocimiento (Guastini, 2018, capítulo V, II).

8 Esto no significa necesariamente que estos dos tipos sean exhaustivos del conjunto de las normas. Cfr. (Comanducci, 1998, p. 90).

${ }^{9}$ Que puede ser atribuida, al menos en algunas lecturas, a H. Kelsen. Cfr. (Alonso, 2010, p. 201). Véase la nota 16 para el problema de la derrotabilidad ( defeasibility»).

10 El mismo Alexy afirma que existen tres tesis: i) no diferenciación por no posibilidad de distinguir entre estos; ii) diferenciación de grado; iii) diferenciación cualitativa (1993, p. 85). Cfr. (Alonso, 2010; Comanducci, 1998).

${ }^{11}$ Cfr. J.P. Alonso (2010, p. 202), siguiendo a R. Dworkin (1989, pp. 61-ss).

${ }^{12}$ Cfr. (Alexy, 1993, pp. 98-101). Este autor también agrega una diferencia en cuanto al «diferente carácter prima facie» de las reglas y de los principios, criticando a Dworkin.

13 Cfr. J.P. Alonso (2010, p. 202), siguiendo a R. Dworkin (1989, pp. 61-ss).

${ }^{14}$ Cfr. (Alexy, 1993, pp. 87-98).
} 
Alexy, por ejemplo, ${ }^{15}$ sostiene que: a) las reglas son órdenes definitivas y los principios son mandatos de optimización; b) las reglas se aplican a-todo-o-nada; los principios se aplican gradualmente, según consideraciones relativas a las posibilidades factuales y normativas; y c) en los conflictos regla-regla, o se introduce una excepción implícita a una de estas o se declara su invalidez; en los conflictos principio-principio, la solución es utilizar la técnica de la ponderación (Alexy, 2010, pp. 21-23). Dworkin, el primer exponente de esta tesis, está de acuerdo: solo hace mayor hincapié en las diferencias estructurales entre reglas y principios, y afirma que la solución a los conflictos principio-principio debe obtenerse tomando en consideración aquello que es requerido por razones de tipo moral (Alexy, 2010, p. 23; Dworkin, 1989, pp. 61-ss).

\subsubsection{La TDD}

Los autores que sostienen la TDD critican a la TDF bajo diversos aspectos. Fundamentalmente, sostienen que los principios y la reglas no tienen una calidad, estructura u ontología diferente sino que comparten las mismas características, aunque: i) las tengan en diferente grado y/o ii) cumplan funciones diferentes en el marco del sistema jurídico.

En general, la característica que consideran común, pero presente en diferente grado, es la especificidad o determinación de cada norma. En este sentido, sostienen que las reglas y los principios tienen la misma estructura lógica: ${ }^{16}$ ambos son enunciados que correlacionan un antecedente con un consecuente. ${ }^{17} \mathrm{La}$ diferencia residiría entonces en el hecho de que, mientras que las reglas tienen antecedentes y consecuentes muy precisos ("cerrados"), los principios tienen antecedentes y consecuentes muy vagos e indeterminados ("abiertos"), a partir de los cuales no pueden identificarse prima facie ni los casos en los cuales estos se aplican ni las soluciones normativas concretas para estos. ${ }^{18}$

Así, puede decirse que la TDD sostiene que:

1) Principios y reglas no son ontológicamente diferentes;

2) Principios y reglas tienen la misma estructura lógica, y las diferencias son relacionales y/o graduales; $y$

3) El hecho de que una norma sea un principio o una regla es meramente contingente, y depende de una decisión del juez o del intérprete; en otras palabras, sostiene que es falso que una norma sea, original y netamente, una regla o un principio (Comanducci, 1998, p. 94).

Los principios aparecen, por tanto, como normas más genéricas, indeterminadas y/o jerárquicamente superiores en relación con las reglas. Por ejemplo, C. Alchourrón y E. Bulygin sostienen que los principios son simplemente generalizaciones obtenidas a partir de un conjunto de reglas (Alchourrón \& Bulygin, 2015, pp. 123-124). P. Comanducci, por otra parte, sostiene que la diferencia es relacional y jerárquica: en un determinado momento, una norma superior $\mathrm{N} 1$ puede ser configurada como principio respecto a normas inferiores,

\footnotetext{
15 Para una mirada de otros autores que sostienen la TDF, pero con una definición tripartita, cfr. (Atienza \& Ruiz Manero, 2007).

${ }^{16}$ Normas hipotéticas.

${ }^{17} \mathrm{El}$ antecedente es un conjunto de propiedades relevantes, cuyo acaecimiento es necesario para volver aplicable una norma; el consecuente es, en cambio, la consecuencia normativa que debe seguirse una vez acaecido el antecedente.

${ }^{18}$ Un problema particular es el de la derrotabilidad («defeasibility») de las reglas, i.e. que estas puedan no ser aplicables a un caso concreto por excepciones no explicitadas en sus antecedentes. Si se admite la derrotabilidad de las reglas, entonces la idea del antecedente cerrado como diferencia entre reglas y principios parece caer. Abordaré esta problemática en otra sede, limitándome aquí a seńalar su existencia. Para una síntesis de esta problemática, cfr. (Alonso, 2010, pp. 211-213).
} 
configuradas como reglas; pero, en otro momento, N1 puede ser configurada como regla respecto de una norma N2 superior a esta (Comanducci, 1998, pp. 97-ss).

\section{Normas de derechos humanos: ¿principios o reglas?}

3.1. La teoría: ¿̨reconstrucción como principios o reglas?

\subsubsection{Rechazo de la TDF}

Comparto la opinión ${ }^{19}$ de aquellos que sostienen que la TDF debe ser, por razones conceptuales, rechazada. ${ }^{20} \mathrm{Y}$ esto, sobre todo, por dos motivos: por un lado, por aquello que la TDF asume respecto de la naturaleza de las normas; por el otro, por las tesis que la TDF contiene acerca del modo de aplicación de reglas y principios.

En relación con el primer motivo, concuerdo con Comanducci cuando señala que el considerar a la TDF como una tesis teórica no parece superar el test de verificación empírica, respecto de las características atribuidas a los principios y a las reglas (Comanducci, 1998, p. 95). Además, el mismo test de verificación empírica podría también mostrar que la "identificación” de una norma como regla o principio depende de la práctica de una comunidad jurídica de referencia. Allí, de forma contingente, puede existir una práctica establecida y aceptada de identificar a las normas (por ejemplo) sobre derechos humanos como principios. Sin embargo, nada impide -jurídicamente, como mínimo, pero sin duda también socialmente- que en el futuro esta práctica pueda cambiar.

En relación con el segundo motivo, hay dos críticas fuertes relacionadas con el modo de aplicación de reglas y principios: 1) no es verdad que, en un conflicto reglaregla, necesariamente se deba declarar la invalidez de una de estas; y 2) es incompatible afirmar que i) las reglas tengan una aplicación a-todo-o-nada (conociéndose desde el inicio, por tanto, todas sus posibles excepciones) y que, al mismo tiempo, ii) las reglas puedan ser "excepcionadas" por los principios (de los cuales no se conocen ab origine todas las excepciones posibles). ${ }^{21}$

Por estos dos motivos, entiendo que las TDD permiten entender mejor el fenómeno de las normas en general, y de las normas de derechos humanos en particular, especialmente en lo que respecta a las diversas dificultades relacionadas con su aplicación e interpretación, además de su uso en el razonamiento jurídico. ${ }^{22}$

\subsubsection{La estructura lógica de los derechos humanos}

Ahora bien, se puede decir que generalmente las normas de derechos humanos son consideradas como principios por dos razones: 1) la indeterminación que las caracteriza; y 2) el rol que cumplen en el razonamiento jurídico (un rol "fundamental"). ${ }^{23}$

En relación con el antecedente, la mayor parte de los derechos humanos, así como han sido positivizados, no identifica precisamente los casos a los cuales se aplican: esto es, tienen un antecedente abierto. De esta forma, dejan al intérprete la decisión de si,

\footnotetext{
${ }^{19}$ Sostenida, por ejemplo, por P. Comanducci (1998) y J.P. Alonso (2010).

${ }^{20}$ Por razones de espacio, aquí sostendré de modo axiomático el rechazo de la posición de la no diferenciación.

${ }^{21}$ Cfr. (Alonso, 2010, p. 175), citando a J.C. Bayón (1991).

22 Esta también es la opinión de P. Comanducci (1998). Hacer propia esta posición, sin embargo, no significa afirmar sin más que los derechos humanos sean principios y no reglas: significa solamente afirmar que una TDD permite hacer un mejor análisis del estatus de los derechos humanos (en particular, si estos deban ser considerados principios o reglas), también considerando las características particulares que normalmente se atribuyen a estas normas.

${ }^{23}$ De hecho, estas dos características son aquellas identificadas por R. Guastini en su caracterización de los principios. Cfr. (Guastini, 2018, p. 218).
} 
en un determinado caso específico, se encuentran o no presentes las propiedades relevantes necesarias para volver invocable al derecho humano en análisis. ${ }^{24}$

En relación con el consecuente, sin embargo, este generalmente no es completamente indeterminado: ${ }^{25}$ adscribe consecuencias normativas del tipo "facultativo $X$ " o "obligatorio proveer $X$ " o "prohibido interferir en $X$ ”. Estas consecuencias pueden ser indeterminadas en relación con cuáles sean las conductas que deben ser materialmente realizadas (para proveer o para no interferir), pero no son igualmente indeterminadas en relación con la obligación o la prohibición dispuestas.

En definitiva, es posible afirmar que, si se conciben a las normas de derechos humanos como principios, su estructura sería antecedente abierto/consecuente cerrado. ${ }^{26}$

\subsection{Más allá de la teoría: ¿̨cómo considerar a las normas de derechos humanos?}

Dar una respuesta a esta pregunta tiene mucho que ver con, por ejemplo, consideraciones ideológicas (Alexy, 2010, p. 21). La elección entre considerar las normas de los derechos humanos ${ }^{27}$ como reglas, como principios, o como ambos, se transforma de una cuestión de hechos -como pueden ser entendidos los derechos humanos- a una cuestión de valores -como deben ser entendidos, según la función que estén llamados a desempeñar en el ámbito del derecho, según una determinada ideología-.

Considerar a los derechos humanos como reglas implica concebir a los tratados en materia de derechos humanos como espacios rígidos pero delimitados, un ámbito reservado (coto vedado) ${ }^{28}$ en el cual los derechos humanos se consideran atrincherados, para su protección. Las condiciones de aplicación son muy claras y no extensibles, y las consecuencias normativas son fácilmente identificables para todos los casos allí comprendidos. En este sentido, los derechos humanos funcionarían de modo todo-o-nada: una vez identificado un caso susceptible de ser subsumido en alguno de estos derechos, la norma correspondiente sería aplicada sin considerar otras circunstancias factuales o normativas. Cualquier otro caso, no subsumible, no podría dar lugar a la aplicación de estas normas ni, por tanto, de los derechos humanos. De esta manera, los derechos humanos podrían ser considerados como derechos en sentido "estándar", esto es, algo que puede reivindicarse, pues la determinación de su contenido no sería (completamente) dependiente de la voluntad del intérprete.

La concepción (o modelo) de los derechos humanos como reglas, en principio, parecería tener dos problemas. Por un lado, la así llamada aplicación todo-o-nada implicaría la realización de una ponderación preliminar, así como sucede cuando los derechos humanos son considerados principios (y, por tanto, concretizados para poder ser aplicados). Por otro lado, e incluso si se rechaza la conclusión precedente relativa a la ponderación preliminar, permanece la circunstancia de que las normas de derechos humanos son el producto de una actividad interpretativa: y, por lo tanto, dependientes de un acto de decisión del juez/intérprete.

\footnotetext{
${ }^{24}$ Aceptando que sea necesario concretizar los principios antes de su aplicación, puede afirmarse que el intérprete tiene que tomar dos decisiones: la primera en relación con las propiedades relevantes que componen el antecedente (para así "cerrarlo" y establecer el caso genérico de la norma); la segunda, en relación con el juicio positivo o negativo de subsunción del caso particular en el caso genérico. Sobre la concretización de los principios, véase el punto 4 del presente trabajo.

${ }^{25}$ Acerca de esta línea de pensamiento, cfr. (Atienza \& Ruiz Manero, 2007).

26 (Atienza \& Ruiz Manero, 2007).

${ }^{27}$ En adelante, por cuestiones de brevedad, abreviaré la expresión y usaré “derechos humanos" para referirme tanto a la noción en abstracto como a las normas de derechos humanos en concreto.

${ }^{28}$ Utilizando terminología de E. Garzón Valdés. Para un desarrollo de este concepto específico, véanse por ejemplo (Garzón Valdés, 1989, 2000).
} 
Considerar a los derechos humanos como principios, en cambio, delinea un modelo más cercano a las doctrinas del neoconstitucionalismo (Pietro Sanchís, 2004), en las cuales los tratados de derechos humanos no son simplemente corpus normativos aislados de los diferentes sistemas jurídicos y/o colocados en una posición jerárquica superior y aislada. En realidad, según esta perspectiva, estos derechos-principios atraviesan trasversalmente a todas las normas, constitucionalizando el sistema y los contenidos jurídicos, además de la interpretación que se haga de estos. La caracterización de los derechos humanos como principios volvería así posible su así llamado efecto horizontal: esto es, que puedan ser usados en cada parte del sistema jurídico de referencia, como máxima de interpretación de todas las relaciones allí contenidas. Los conflictos no serían una cuestión de aplicación-o-no drástica, sino de ponderación.

Sin embargo, esta segunda concepción no está exenta de problemas. El problema más evidente es el hecho de que, considerados como principios (cuyo antecedente, así, no es preciso), los derechos humanos no podrían -en cuanto tales- formar parte del razonamiento judicial, entendido como silogismo. ${ }^{29}$ Para superar tal obstáculo, hay al menos dos alternativas: o bien los derechos humanos son entendidos como "guías interpretativas" para elegir una determinada regla ${ }^{30}$ que cumpla la función de premisa mayor del razonamiento; o bien se recurre a la "transformación" de los principios en reglas, es decir, una "transformación" que los vuelva más específicos o más concretos.

Cualquiera de las alternativas precedentes podrían sin embargo, según algunos autores, abrir la puerta a la discrecionalidad judicial en sentido fuerte, ${ }^{31}$ a la arbitrariedad y/o volvería imposible el control racional de las decisiones de los tribunales (Moreso, 2006); y todo esto podría resultar claramente dańoso en lo que respecta a la protección y garantía de los derechos humanos.

\section{Una vía intermedia: principios entre concretización y regularización \\ 4.1. Concretización}

Como fue dicho anteriormente, es posible afirmar que los derechos humanos son principios que, sin embargo, para poder ser considerados como aplicables a un caso, deben ser "especificados" o "concretizados" (ya sea por el legislador infra-constitucional como por el juez al momento de la decisión). Esto en cuanto su indeterminación -especialmente, aquella de su antecedente- no les permite funcionar como premisa normativa de un razonamiento judicial (Guastini, 2018, p. 244).

Esto es particularmente evidente no sólo en el ámbito internacional, con los diferentes tratados de derechos humanos (como la DUDH), sino también en el ámbito nacional, con las diversas normas constitucionales que establecen derechos fundamentales. En este caso, «los casos genéricos que regulan son necesariamente muy amplios y, por lo tanto, la reconstrucción -el paso de los principios a las reglas- se hace imprescindible» (Moreso, 2006, p. 25).

La concretización de un principio "es un razonamiento -usualmente, no deductivo $y$, por ende, no vinculante- que (a) tiene entre sus premisas al principio en cuestión y (b) tiene como conclusión una regla (inexpresa)" (Guastini, 2018, p. 247). Esta regla inexpresa, que

\footnotetext{
${ }^{29}$ Sobre el razonamiento judicial, véase la bibliografía citada en (Guastini, 2018, p. 283, nota 12).

30 Se hace aquí referencia a 'regla' en sentido estricto, y no a 'regulación' en sentido amplio, porque el punto es señalar que solo las reglas así entendidas pueden ser utilizadas como premisas mayores/premisas normativas de un razonamiento de este tipo.

${ }^{31}$ Según la terminología de R. Dworkin; cfr. (Dworkin, 1989, pp. 61-ss).
} 
tiene un antecedente relativamente determinado o cerrado, será adecuada para ser utilizada como premisa normativa en un razonamiento judicial.

En el caso en el cual haya un conflicto entre principios, este razonamiento incluirá una ponderación -la fijación, esto es, de una jerarquía entre estos, que permita aplicar uno y no el otro- cuya conclusión será, justamente, una regla inexpresa. El método consta de tres pasos:
1) Realizar una ponderación (esto es, fijar una jerarquía);
2) Extraer de esta ponderación una regla (inexpresa); y
3) Evaluar la posible subsunción del caso particular en análisis en la regla obtenida en el paso $2 .^{32}$

Sin embargo, dado el carácter que generalmente se les atribuye a las jerarquías producidas con tal ponderación -esto es, carácter axiológico y móvil-, y dada la caracterización que se puede hacer de tales conflictos entre principios -antinomias en concreto de tipo parcial bilateral-, esta regla inexpresa resultante podría ser considerada como adecuada para regular únicamente ese caso particular en análisis (Guastini, 2018, pp. 249-250). Esto significaría adoptar una concepción particularista y, en este sentido, puede dudarse de su idoneidad para afrontar las críticas desarrolladas en el punto 3.2.

\subsection{Regularización}

Una forma posible de reducir este particularismo señalado en los casos de conflictos entre principios es a través de la así llamada "regularización" (Moreso, 2006, p. 22).

La "regularización" tiene tres diferencias importantes con respecto al método anterior: i) no considera a la jerarquía establecida por la ponderación como completamente "móvil", ya que ii) la regla inexpresa resultante debe ser precisa, es decir, tener antecedente y consecuente claros y cerrados (debe, por tanto, seleccionar todas las propiedades relevantes que estructuran el ámbito normativo del supuesto de hecho o caso); y, en consecuencia, iii) luego de un caso donde se haya seguido el método mencionado y, por tanto, haya sido establecida una regla, el nuevo caso en análisis no debe ser considerado como implicando un conflicto entre principios -el cual requeriría una nueva ponderación- sino como un caso regulado por esta regla. ${ }^{33}$

De esta forma, la regularización transforma también el análisis: de una concepción particularista a una concepción más sustantiva o universalista (Moreso, 2006, p. 24), manteniendo la idea de que las normas de derechos humanos sean consideradas principios y, al mismo tiempo, reduciendo las críticas señaladas en el punto 3.2.

\section{Conclusión}

El presente artículo ha analizado brevemente las normas de derechos humanos y, principalmente: 1) las nociones de regla y de principio; 2) las evaluaciones que considero que se encuentran subyacentes a una y otro, junto con sus correspondientes problemas y críticas. Finalmente, ha sido propuesto un modo posible de volver compatibles: i) la visión de las normas de derechos humanos como principios (siendo así indeterminadas y fundamentales); ii) una concepción no (completamente) particularista de los principios y, por tanto, de los

\footnotetext{
32 Este último paso es la continuación del razonamiento: los pasos 1 y 2 se refieren a la elección de la premisa mayor.

33 A menos que no se sostenga que haya una propiedad relevante previamente no considerada como tal y, por tanto, que la regla ya obtenida no deba ser aplicada: es decir, que exista una laguna axiológica. Cfr. (Alchourrón \& Bulygin, 2015, pp. 157-ss). Sin embargo, tal conclusión debe ser justificada por el juez.
} 
derechos humanos así considerados; y iii) la atenuación de aquellos elementos que pueden resultar dańosos para la protección y garantía los derechos humanos.

Adoptar esta posición, como he sostenido arriba, volvería posible, por un lado, ilustrar más adecuadamente aquello que sucede en la práctica jurídica. Por el otro, permite realizar un análisis racional más preciso y profundizado en relación con las decisiones judiciales que tienen como objeto uno de estos derechos, para poder así garantizar -de la forma más amplia e igualitaria posible- los derechos humanos.

\section{Referencias bibliográficas}

Alchourrón, C. E., \& Bulygin, Eugenio. (2015). Sistemas normativos. Introducción a la metodología de las ciencias jurídicas (2da ed.). Astrea.

Alexy, R. (1993). Teoría de los derechos fundamentales (E. Garzón Valdés, Trans.). Centro de Estudios Constitucionales.

Alexy, R. (2010). The Construction of Constitutional Rights. Law and Ethics of Human Rights, 4(1), 21-32. https://doi.org/10.2202/1938-2545.1042

Alonso, J. P. (2010). Interpretación de las normas y derecho penal (1st ed.). Editores del Puerto.

Atienza, M., \& Ruiz Manero, J. (2007). Las Piezas del Derecho: Teoría de los enunciados jurídicos. Ariel.

Bayón Mohino, J. C. (1991). La Normatividad del derecho: Deber jurídico y razones para la acción. Centro de estudios constitucionales.

Comanducci, P. (1998). Principios jurídicos e indeterminación del derecho. Doxa. Cuadernos de Filosofía del Derecho, 21-v2, 89-104. https://doi.org/10.14198/ DOXA1998.21.2.07

Dworkin, R. (1989). Los derechos en serio. Ariel.

Garzón Valdés, E. (1989). Algo más acerca del «coto vedado». Doxa. Cuadernos de Filosofía del Derecho, 6, 209. https://doi.org/10.14198/DOXA1989.6.12

Garzón Valdés, E. (2000). El consenso democrático: Fundamento y límites del papel de las minorías. Isonomia, 12, 7-34. http://www.scielo.org.mx/pdf/is/n12/1405-0218is-12-00007.pdf

Guastini, R. (2018). Interpretar y argumentar. Ediciones Legales.

Moreso, J. J. (2006). Dos concepciones de la aplicación de las normas de derechos fundamentales. Revista Direito GV, 2 (2), 13-30. http://bibliotecadigital.fgv.br/ojs/ index.php/revdireitogv/article/view/35139/33908

Pietro Sanchís, L. (2004). El constitucionalismo de los derechos. Revista Española de Derecho Constitucional, 71, 47-72. https://recyt.fecyt.es/index.php/REDCons/article/view/48325

\section{Convenciones, tratados y conferencias}

ONU (1948). Declaración Universal de Derechos Humanos: DUDH. Adoptada y proclamada por la Asamblea General el 10 de diciembre de 1948. 